\title{
Port Management and Operations: Emerging Research on Hotspots and Their Progress
}

\author{
Mingqi Zhang \\ Tianjin Maritime College, Tianjin, 300350, China
}

Key words: port management and operation; research hotspots; research progress

\begin{abstract}
With the development of the national strategy "the Belt and Road Initiatives ", that have brought new opportunities and challenges to China's port management and operation. Based on the research results of port management and operation at home and abroad, this paper firstly analyzes the shortcomings and potential emerging research hotspots in China's port management and operation, and then analyzes the progress of port management and operation.
\end{abstract}

\section{Introduction}

Port is the most important part of marine transport, from the research data show that $80 \%$ of the material trade is completed through the port and its connected marine transport line. In addition, the total amount of marine transport is also rising, the total transport volume in 2014 reached 9.84 billion tons, and China's port container throughput in 2014 also accounted for $25 \%$ of the global port throughput, our country has seven of the world's top 10 container ports [1]. It can be seen that the study of port management and operation is very important. Since 2014, China has also achieved theoretical and practical achievements in port management and operation research. Many research projects have received support from state funds, the most important of which are: multi-resource configuration optimization, port-to-port collaboration issues, and transportation network optimization. The potential research focus on port management and operation which mainly includes the optimization of the operational resources on the operational layer decision-making and the optimization of the integration of the port group service resources, the optimization of the port group multi-mode transportation network at the tactical level, and the collaboration between the ports at the strategic level behavior and mechanism operations, collaboration and mechanisms between ports and key stakeholders.

\section{Decision-Making of Port Management and Operation}

\section{1 decision-making of operational management}

The emerging hot issues that need to be studied deeply in the operational decision-making are mainly the online optimization of operational resources and the optimization of the integration of single port or port group resources. In the past, the resource optimization problem of single port was focused on the single resource, but the emerging research hotspot began to shift to the optimization of multi-resource integration. In addition, with the emergence of the theory of robust operation and real-time optimization of operation, the scholars also turn ed the study hotspot to more practical orientation and close to the modern port, enhance the modern port operation ability research for the operation optimization and let it become the focus of future research.

\subsection{Tactical decision making}

From the tactical point of view, the port group dimension transport network design and the choice of the relevant transport mode has become a hot topic in this field. The design of the port transport network is mainly based on the specific target, around the relevant resource elements, such as fleet, boat line, service pricing and speed setting, route frequency and other network integration design research. The main research in the choice of transport mode is mainly based on the optimization of transport network design, and the comparison between the various transportation modes in different modes of transportation. 


\subsection{Strategic decision-making}

The focus of further research at the strategic level is the collaboration and mechanisms between ports, as well as the collaboration and mechanisms of key stakeholders in the port. The research on cooperation and mechanism between ports is mainly related to the analysis of the key elements and the relationship between port group and port group collaboration, and the theory of game competition and case analysis is applied to the research in this field. The main study of the major port stakeholder collaboration and mechanism is the choice of ports in the maritime supply chain, the way in which the port is in line with the carrier, and the relationship between the port carrier and the shipper. In this regard, the emerging research hotspot is from the overall macro perspective, the study of maritime supply chain overall competition and cooperation [2].

\section{Related Research Analysis on Progress of Port Management and Operation}

\subsection{The studies of the single port dimension consist of three main aspects}

First, the single resource allocation problem of single port, single resource allocation research mainly involves berth allocation research, shore bridge scheduling research and yard management. For example, in the allocation of berths, scholars have studied the types of berth models. The types of berths are mainly discrete berths, continuous berths and mixed berths. The essence of the former is the parallel machine scheduling problem, and the latter is two-dimensional packing problem. Lalla-Ruiz [3] and others have proposed the influence of water depth and tidal effect on berth in the study of berth allocation model, in recent years the study of berth allocation on uncertain environment has also become a hot issue. For example, Zhen [3] has proposed the problem of parking in discrete berth model, with the features of uncertainty service and periodicity, and constructed a robust optimization model, finally the model is built and analyzed. Followed by the quayside scheduling, the quayside dispatch is in the berth after the distribution, according to the container loading and unloading capacity for the ship which can be used to allocate the quayside, and then make a detailed distribution plan to allocate the bridge. In the study of the quayside scheduling, Zhen [3] analyzed the difference between the quayside scheduling and the parallel machine scheduling. In addition, according to the hypothesis that the quayside can only move in a single direction, the model of Al-Dhaheri [3] and his colleague had used the genetic algorithm to analyze the quay scent bridge scheduling model based on the factors such as the movement time of the bridge and the weight distribution of the container. In addition, the purpose of yard management research is to allocate the yard space reasonably and improve its utilization rate. Its essence is the problem of four-dimensional packing. On the basis of the study of yard management, Wei Hang studied the time window optimization model of containerized container loading in consideration of reducing the number of port rollovers. Zhen [3] and others have put forward a mixed model of multi - stage storage space allocation and designs the algorithm to solve the large - scale model based on that different freighters has different period. In addition, due to the study of various fields in uncertain environment in recent years, Zhen and others had studied the flexible resource allocation of yard space allocation under the uncertain conditions of cargo ship parking time and location. In short, the research on the single resource allocation problem of single port has been relatively mature, the research system has gradually improved, and the problem of multi-resource integration allocation for single port will become hot. At present, the integration of resource optimization is mainly the integration of two kinds of resources, such as the allocation of berth and the integrated scheduling of the quayside, the coordinated dispatching of the bank and the card, the integration of the berth and the yard. In the berth and shore-bridge integration scheduling, some scholars have constructed a three-stage integrated optimization framework according to the coordination problem with berths and quayside. Vacca [3] and others use the column generation method to model the berth and the quayside and carry out the accurate model calculation. In the optimization of berths and yard, there are scholars have studied the problems with the transport port continuous berth and the coordination of the optimization. Finally, the research on the optimization of resource online has become a hot spot, and a lot of research results have been made in flight planning, logistics and 
distribution. Zhen and others have constructed a resource optimization strategy based on robust optimization.

\subsection{The progress of the research on port group dimension can also be divided into three parts}

First, the port cluster services resource allocation optimization is based on the specific port group network structure and related constraints, and consider the relationship between supply and demand between ports, constructed a flexible resource allocation plan. Erera [4] and others have constructed a robust optimization model in space-time networks, proposed robustness to the three network flows and proposed uncertain sets to solve them. In this study, $\mathrm{Yu}$ and others used the theory of game competition to construct the monopoly and duopoly model, and put forward their own views on the carrier's optimal pricing strategy. Follow by the study of port group transport network optimization. Transport network designs can be divided into non-scheduled service network design and liner service network design. But at present, most of the study is liner service networks to design, such as Gelareh [4] and others in the liner network design model has considered the transport costs. At present, there are some progresses of the study of fuel consumption and uncertain factors. For example, Xia [4] and others have considered the nonlinear fuel consumption function as this model study. Finally, the study on the cooperative behavior and mechanism between the port groups, such as Kim [4] and others have studied and put forward relevant strategies on the China's ports and other countries around the port competition between the status quo from the port group competition . Such as Low [4] and others proposed the theory of Central Asian container port group network effect.

\subsection{Progress of Research on Main Stakeholders Relationship between Port and Sea Supply Chain}

The research on the relationship between the main stakeholders of the port and the sea supply chain refers specifically to the research on the cooperative behavior and mechanism between the carrier and the maritime supply chain of the carrier. Such as Tang and others have built a model of comprehensive port selection, and through the analysis of the shipping company has selected the port to consider the main factors. Tang and others have constructed a decision-making model based on service pricing, and analyzed various factors. Some scholars have focused on the selection relationship between the carrier and the port. Lee and others have analyzed the roles and functions of the stakeholders in the maritime supply chain and the competitive relationship between them, and the influence of the port throughput on the choice of supply chain of the sea.

\section{Conclusion}

In summary, the current port management and operation has achieved good results from a single port single resource allocation optimization research, the research in a comprehensive environment optimization of a variety of resources, and the problem of port group service resource allocation optimization and transportation network design, and the research on the uncertain environment of a variety of resource integration optimization, the research on how to obtain accurate solution on the large-scale model, as well as the research of the competition and cooperation on the supply chain will become potential hot research issue .

\section{References:}

[1] Yang Chao, Lu Zhiqiang.Optimization of time window of container delivery in export container based on genetic algorithm [J] .Application Research of Computers, 2013 (6): 1643-1646.

[2] Liu Di, Yang Hualong, Zhang Yan. Multi-node container sea-iron linkage dynamic pricing decision [J]. Journal of Systems Engineering and Practice, 2014 (1): 104-114.

[3] Xu Lei, Bu Xiangzhi, Feng Lipan, Li Chunfa. Shipping service chain in the capacity of the pricing and empty transport responsibility of the study 
http://60.191.152.123:85/article/detail.aspx?id=662722992 [J]. With management, 2014 (5): 101-108.

[4] Zhang Yuting, Huang Youfang, Yan Wei. Container Terminal Yard Functional Partition and Simulation http://60.191.152.123:85/article/detail.aspx?id=663326067 [j]. Shanghai Maritime University, 2014 (4) : 37-43. 